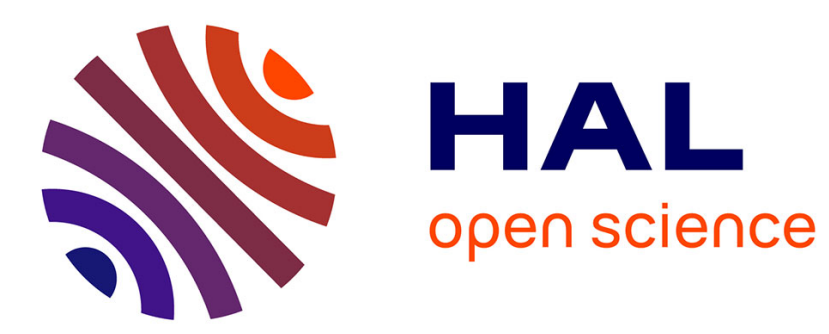

\title{
Depth control of the biomimetic U-CAT turtle-like AUV with experiments in real operating conditions
}

\author{
Ahmed Chemori, Keijo Kuusmik, Taavi Salumae, Maarja Kruusmaa
}

\section{To cite this version:}

Ahmed Chemori, Keijo Kuusmik, Taavi Salumae, Maarja Kruusmaa. Depth control of the biomimetic U-CAT turtle-like AUV with experiments in real operating conditions. ICRA: International Conference on Robotics and Automation, May 2016, Stockholm, Sweden. pp.4750-4755, 10.1109/ICRA.2016.7487677 . lirmm-01343321

\section{HAL Id: lirmm-01343321 \\ https://hal-lirmm.ccsd.cnrs.fr/lirmm-01343321}

Submitted on 9 Sep 2019

HAL is a multi-disciplinary open access archive for the deposit and dissemination of scientific research documents, whether they are published or not. The documents may come from teaching and research institutions in France or abroad, or from public or private research centers.
L'archive ouverte pluridisciplinaire HAL, est destinée au dépôt et à la diffusion de documents scientifiques de niveau recherche, publiés ou non, émanant des établissements d'enseignement et de recherche français ou étrangers, des laboratoires publics ou privés. 


\title{
Depth Control of the Biomimetic U-CAT Turtle-Like AUV With Experiments in Real Operating Conditions*
}

\author{
A. Chemori ${ }^{1}$, K. Kuusmik ${ }^{2}$, T. Salumäe ${ }^{2}$ and M. Kruusmaa ${ }^{2}$
}

\begin{abstract}
Control of underwater vehicles is a thoroughly investigated subject but still an open problem, because of the environmental disturbances, the highly nonlinear behaviour of vehicles, the complexity of the vehicle hydrodynamics, etc. In this paper, we are interested in depth control of a bioinspired U-CAT underwater AUV in real operating conditions. Two depth control schemes are proposed, including a PID controller and a nonlinear RISE feedback controller. The proposed controllers are implemented on the robot, then tested in an open water environment. The obtained results are presented and discussed through different experimental scenarios to illustrate the efficiency of the proposed controllers, not only to successfully control the depth, but also to be robust towards external disturbances and parameters uncertainties. we conclude that RISE controller is more robust towards environmental disturbances and outperforms the PID controller when the robot is tested in real operating condition.
\end{abstract}

\section{INTRODUCTION}

Biomimetic underwater robots propose alternatives for conventional propeller-driven underwater vehicles. Median and paired fin (MPF) locomotion is usually suggested as a viable alternative if high maneuverability and hovering capability is required. In fishes, such a propulsion mechanism usually means lower speeds (as opposed to body and caudal fin propulsion) but is advantageous when low speed and precision maneuverability is desired [1]. A particular type of MPF propulsion is sea turtle like 4-fin locomotion. Attempts to copy the locomotion of those agile and versatile reptiles reach back at least a decade with "Turtle 2005" [2] and Madeline [3]. Other examples include Finnegan, the RobotTurtle [4] and iRobot Transiphibian, developed by the same author [5]. Another line of development is represented by AQUA [6] and AQUA2 [7] four finned amphibian robots that are unique in the way the propulsors are used both for swimming and crawling in and out of water. [8] describes a four-finned robot with a controllable fin surface and the authors in particular focus on optimizing the performance of ribbed fins by actively changing the surface contour [9], [10]. Four finned propulsion is also realized by deploying a scaffold structure actively controlled by shape memory alloy (SME) wires [11]. This paper describes an autonomous underwater robot U-CAT that is developed in European

*This work was supported within ARROWS European project and PHCPARROT French-Estonian joint collaboration project.

${ }^{1}$ A. Chemori is with the LIRMM UMR CNRS/Univ. of Montpellier, 161 rue Ada, 34392 Montpellier, France, ahmed.chemorielirmm.fr (supported by MAEDI \& MENESR ministries)

2 K. kuusmik, T. Salumäe and $M$. Kruusmaa are with the Centre of Biorobotics, Tallinn University of Technology, 12618, Tallinn, Estonia Keijo.Kuusmik Traavi.Salumae, Maarja.Kruusmaa\}@ttu.ee
Union 7th Framework project ARROWS (Archeological Robot Systems for the World Seas) [12]. As opposed to the previous examples, four-finned design of this vehicle is motivated solely by the end-user requirements and environmental constraints of the tasks in this, specifically shipwreck penetration. As part of the team of ARROWS robots, it would closely video-inspect underwater objects. U-CAT can be operated in a tethered mode through an onboard camera feedback or autonomously, in which case it would enter a construction though an opening, inspect the space with a reflexive obstacle avoidance algorithms, return to the opening using a hydrophone array detecting a pinger and bringing back video footage from the interior of the shipwreck or any other construction. Currently, the robot prototype is undergoing field testing and continuous development of the autonomous capabilities (obstacle avoidance, navigation, localisation).

Control of underwater vehicles is a thoroughly investigated subject but still proposes difficulties, among the other things, because of environmental disturbance, highly nonlinear behaviour of vehicles, complexity of the vehicle hydrodynamics etc [13]. Biomimetic underwater vehicles usually control motion by changing the locomotion primitives of the fins, usually frequency, amplitude, phase shift, or in more complicated cases also the angle of attack, stiffness or surface area. Previously, MPF actuated 4 finned robots have been controlled using adaptive control [7], PID control [8] but also bio-inspired CPG control [14]. U-CAT uses 1 DOF flexible fins but as opposed to the previously cited work, the fins are placed in corners of the vehicle under an angle so that the thrust vectors created by each individual fin are not collinear. All 4 fins are independently driven, making the robot holonomic. As opposed to a propeller driven equivalent, such a finned design proposes additional difficulties due to its delayed response and complex hydrodynamics. For example, if changing the direction of the translational velocity vector (e.g. when stabilizing or hovering) the fins have to turn 180 degrees which take finite time and creates hydrodynamic reaction forces from the environment.

In this paper we develop and test two non-model-based one-dimensional (depth) control approaches of U-CAT: PID control and RISE feedback control. The PID control is a feasible approach to start with either because it may be sufficient to solve the problem with a satisfactory precision, or, alternatively, because it can serve as a benchmark for comparison with more advanced control approaches (such as the second controller, i.e. the nonlinear RISE feedback control). For instance, [8] reported good results using PID 
heading and depth control. In [7], experiments where run in open dynamic environment with adaptive control, reporting satisfactory roll performance, lag in pitch tracking and also concluding that the adaptive law converged to a value similar obtained in the linearization.

The rest of the paper is organized as follows. The next section shortly describes the U-CAT underwater robot. Then we describe the proposed depth controllers in section III. The obtained experimental results in open water environment with environmental disturbances are presented and discussed in section IV. The paper ends with conclusions and description of our future work directions.

\section{U-CAT Biomimetic Robot Design CONCEPT}

U-CAT is a biomimetic robot (cf. Fig. 1) designed specifically to meet end user requirements of underwater archaeologists. The design principles of U-CAT are laid down in [15] which describes the constraints and specific requirements posed by the nature of ship penetration on an archaeological missions. As a consequence, a 4-flipper design emerged, not because there was a specific aim to design a bio-inspired robot but rather because the biomimetic design was most suitable for solving the problem. Particularly, the crucial requirements where the small size and high maneuverability of the vehicles, that required a fully actuated robot with as few as possible actuators. In U-CAT, 6DOF actuation is realized using 4 motors. Another requirement was quiet motion, that was best realized by flippers. As opposed to the propellers, flippers have a large actuation area and therefore the propelled mass around the robot disturbs bottom sediments less. This makes close video inspection of a ship interior more feasible. The current version of U-CAT,

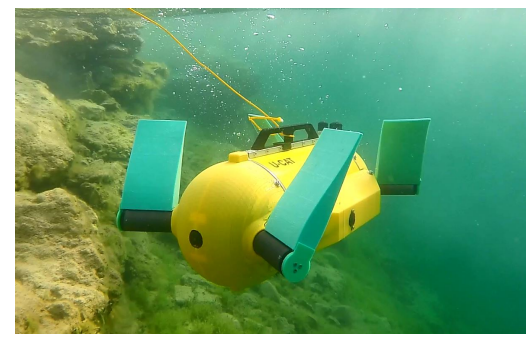

Fig. 1. View of the biomimetic U-CAT AUV operating in an open water environnement, in RUMMU lake (Tallinn, Estonia).

pictured in Fig. 1, is a fully equipped field deployable robotic platform, designed to dive up to $100 \mathrm{~m}$ depth. The $0,6 \mathrm{~m}$ long cylindrical robot houses batteries, electronics, flipper motors and their drivers as well as on board sensors (IMU, hydrophone array, 8 sonars for obstacle avoidance, camera), lighting and modem for underwater communication in untethered mode.

Fig. 2 shows how the 4 independently driven flippers are used to achieve maneuverability in 6 DOF; whereas, Fig. 3 shows the flippers positioning as well as their rotation axes. In addition to the flipper based control, U-CAT also has a buoyancy control unit that gives an additional means for controlling depth, however, depth control in this paper is only

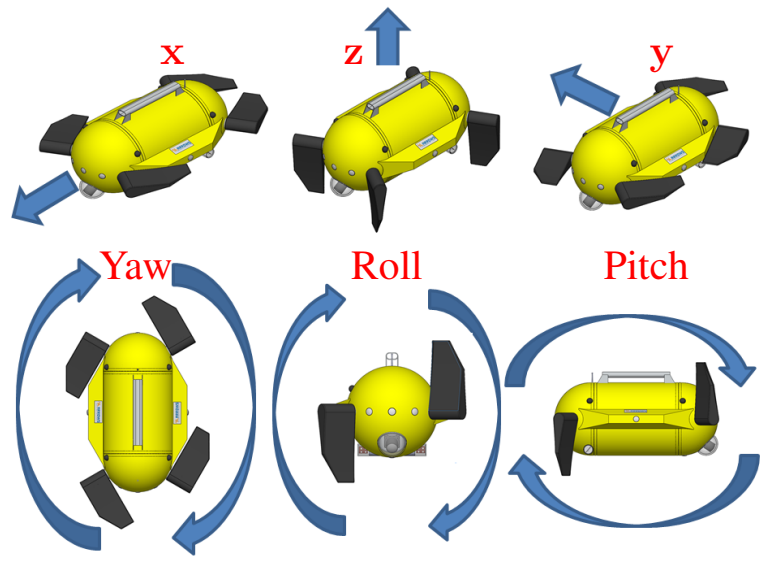

Fig. 2. Actuation of U-CAT: Illustration of how the 4 independently driven flippers are used to actuate the 6 DOF of the robot.

realized by controlling the fins and keeping the buoyancy constant. The robot is also equipped with a depth sensor that in the experiments described in this paper, is used as an output measurement.

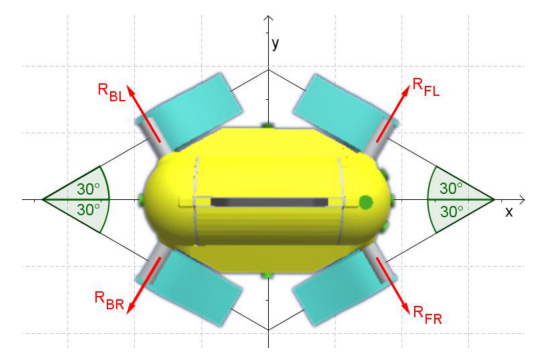

Fig. 3. Flippers positioning and their rotation axes.

\section{PROPOSED CONTROL SCHEMES}

Before going further by introducing the two proposed control schemes, let's summarize first the 6-DOF dynamics of the robot as well as the one-dimensional dynamics of interest to be controlled (i.e depth dynamics).

\section{A. The dynamics of the robot}

By considering the inertial generalized forces, the gravity and buoyancy contributions, the hydrodynamic effects, and the generalized forces generated by the actuators, the hydrodynamic model of an underwater vehicle [16] writes:

$$
\begin{gathered}
\dot{\eta}=J(\eta) \nu \\
M \dot{\nu}+C(\nu) \nu+D(\nu) \nu+g(\eta)=\tau
\end{gathered}
$$

where $\boldsymbol{\nu}=[u, v, w, p, q, r]^{T}, \boldsymbol{\eta}=[x, y, z, \varphi, \vartheta, \psi]^{T}$ represent the vectors of the vehicle velocities in the body-fixed frame and positions in the earth-fixed frame respectively. $J(\eta) \in \mathbb{R}^{6 \times 6}$ represents a transformation matrix, mapping from the body-fixed frame to the earth-fixed frame. The matrices $M, C$, and $D$ denote the inertia including added mass, the Coriolis-centripetal including addeid mass, and the damping respectively. The vector $\boldsymbol{g}$ represents the gravitational/buoyancy forces and moments. The term $\tau$ represents 
the vector of control inputs. Equation (1) describes the dynamic of the system in six DOF, including the three translations and the three orientations. The input vector $\tau \in \mathbb{R}^{6}$ considers six actions on the system to fully control it. Given the available sensors and instrumentation of the robot, we propose, in this work, studying the control of the vehicle in its translational motions along the $z$ axis (depth control). With the assumption of slow dynamics, involving low velocities, the Coriolis terms can be neglected. Therefore, the dynamics (1) can be rewritten as:

$$
M \dot{\nu}+n(\nu, \eta)=\tau
$$

with $n(\nu, \eta)=D(\nu) \nu+g(\eta)$. The hydrodynamic parameters of our robot have been experimentally idientified on UCAT prototype. Since we are interested in depth control, the dynamics of the robot along this direction can easily be extracted from the previous equation and written as:

$$
M_{z} \dot{w}+D_{z} w-\cos (\varphi) \cos (\vartheta)(W-B)=\tau_{z}
$$

The gravitational and buoyancy forces are now brought to a single term which is a combination of $W$ (the weight) and $B$ (the buoyancy). $\tau_{z}$ is one dimensional control input representing the generated force along the $z$-axis and controlling the depth.

In the case of our robot U-CAT, due to the flexibility of the flippers, the control input is a complex nonlinear function depending on three parameters: the orientation, the amplitude and the frequency of oscillations of the flippers, that is:

$$
\tau_{z}=\mathcal{H}\left(\boldsymbol{\Phi}, \boldsymbol{A}_{\text {osc }}, \boldsymbol{f}_{\text {osc }}\right)
$$

where $\mathcal{H}$ is a highly nonlinear function, $A_{\text {osc }}, f_{\text {osc }} \in \mathbb{R}^{4}$ represent the vectors of oscillations' amplitudes (respectively frequencies), and $\Phi \in \mathbb{R}^{4}$ is the vector of orientation angles, around which the flippers will oscillate (an illustration of these orientations to control independently the different DOFs of the robot is shown in Fig. 2). In our case (depth control), the orientation of the flippers is as illustrated in Fig. 2 ( $z$ case, with an orientation upwards or downwards depending on the sign of the depth tracking error). For the case of depth control, the control input $\tau_{z}$ is interpreted as a force (in Newtons). This force produced by the fins has been experimentally measured at a range of different amplitudes given a fixed frequency. Indeed, the frequency of oscillations of the flippers is fixed as well as their orientation, consequently the new control input will be the amplitude of the oscillations. In section IV, the experimental identification of the inverse of the nonlinear function $\mathcal{H}$ is introduced. In the sequel, the two proposed depth control schemes (The PID controller and the RISE feedback control) will be introduced.

\section{B. First Controller : PID control}

The PID is a well known and well established controller [17]. It is the most popular controller in industry, characterized by three feedback gains; namely, a proportional, a derivative, and integral gains.
1) Control Law Formulation: In the case of our robot UCAT, the depth is measured in real-time thanks to a pressure sensor. The controller has to achieve depth control for the tracking of a desired reference trajectory. The control action is given by:

$$
\tau_{z, P I D}=K_{P_{1}}\left(z_{d e s}-z\right)+K_{I_{1}} \int_{0}^{t}\left(z_{d e s}-z\right) d t+K_{D_{1}}\left(\dot{z}_{d e s}-\dot{z}\right)
$$

where $\tau_{z, P I D}$ is the control input, representing the force to be generated by the oscillations of the robot's flippers along the $z$ axis. The parameters $K_{P_{1}}, K_{I_{1}}$ and $K_{D_{1}}$ are positive constants representing respectively the proportional, the integral and the derivative feedback gains. $z_{\text {des }}$ is the desired depth (a time varying trajectory in our case) and $z$ is the measured one; $\dot{z}_{d e s}$ and $\dot{z}$ represent their respective time derivatives.

2) PID Controller Design: The expression of the control input (5) can be reformulated as follows:

$$
u(t)=K_{P}\left[e(t)+\frac{1}{T_{i}} \int_{0}^{t} e(t) d t+T_{d} \frac{d e(t)}{d t}\right]
$$

with $e(t)=\left(z_{\text {des }}-z\right)$ being the tracking error, $K_{P}$ the proportional gain, $T_{i}$ the integral time and $T_{d}$ the derivative time.

\section{Second controller : Nonlinear RISE feedback control}

The RISE (for Robust Integral of the Sign of the Error) [18] strategy is a feedback control technique that yields semiglobal asymptotic tracking, even in the case of presence of uncertainties in the system's dynamics and parameters [19]. This control scheme, using an integral signum term, can accommodate for a wide class of uncertainties based on some assumptions on the disturbance and the controlled system [20].

To quantify the control objective, let's now consider the tracking error $e_{1}(t) \in \mathbb{R}$, and the filtered tracking error $e_{2}(t) \in \mathbb{R}$. These errors are defined by the following:

$$
\begin{gathered}
e_{1}=z_{d}-z \\
e_{2}=\dot{e_{1}}+\alpha_{1} e_{1}, \quad \alpha_{1}>0
\end{gathered}
$$

where $z_{d} \in \mathbb{R}$ represents the desired depth position of the robot. According to [19], the RISE control input can be defined as follows :

$$
\begin{aligned}
\tau_{z, R I S E}= & \left(k_{s}+1\right) e_{2}-\left(k_{s}+1\right) e_{2}\left(t_{0}\right) \\
& +\int_{t_{0}}^{t}\left[\left(k_{s}+1\right) \alpha_{2} e_{2}(\sigma)+\beta \operatorname{sgn}\left(e_{2}(\sigma)\right)\right] d \sigma
\end{aligned}
$$

where $k_{s}, \alpha_{2}, \beta \in \mathbb{R}$ denote positive constant gains and $\operatorname{sgn}(\xi)$ denotes the sign function of $\xi$. Although, RISE feedback control has been applied to various systems, including direct-drive motors [21], hard disc drives [22], propelleractuated AUVs [23], parallel kinematic manipulators (PKMs) [24], etc, to the best knowledge of the authors, it has never been applied to a biomimetic underwater vehicle. 


\section{EXPERIMENTS IN REAL CONDITIONS}

The objective here is to present the experimental results obtained through real-time application of the proposed controllers to U-CAT robot. After some first tests in a swimming pool, the idea was to validate the proposed solutions in real operating conditions in a lake with natural disturbances.

\section{A. Some experimental issues}

The following experimental details are important to underline:

- The parameters of both controllers have been tuned first in a swimming pool; then a fine tuning was performed during the tests in the lake. The obtained parameters are summarized in TABLE I.

\begin{tabular}{|c|c|c|c|}
\hline PID & & RISE & \\
\hline Parameter & Value & Parameter & Value \\
\hline$K_{P_{1}}$ & 10 & $k_{s}$ & 15 \\
\hline$K_{d_{1}}$ & 7 & $\alpha_{1}$ & 0.75 \\
\hline$K_{I_{1}}$ & 5 & $\alpha_{2}$ & 0.5 \\
\hline Windup limit & 0.1 & $\beta$ & 0.5 \\
\hline \multicolumn{3}{|c|}{ windup limit } & 0.1 \\
\hline
\end{tabular}

TABLE I

PARAMETERS OF THE PROPOSED CONTROLLERS AND THEIR VALUES.

- For the investigated case of depth control, the frequency of oscillations is fixed to $f_{o s c}=1.1 \mathrm{~Hz}$ for all the flippers, that is $\boldsymbol{f}_{\text {osc }}=\left[\begin{array}{llll}f_{\text {osc }} & f_{\text {osc }} & f_{\text {osc }} & f_{\text {osc }}\end{array}\right]^{T}$. The orientation of the flippers is also fixed (upwards or downwards, depending on the sign of the depth tracking error). The new control input is the amplitude of oscillation of the fins. Based on the above assumptions and equation (4), the inverse of the nonlinear function $\mathcal{H}$ was experimentally identified, leading to the following relation between the required force and the new control input (amplitude of oscillations):

$$
A_{\text {osc }}=-0.01170\left(\frac{\tau_{z}}{4}\right)^{2}+0.4363\left(\frac{\tau_{z}}{4}\right)+0.2480
$$

This expression gives the oscillation amplitude of one flipper, therefore the vector of oscillation amplitudes will be as follows: $\boldsymbol{A}_{\text {osc }}=\left[\begin{array}{llll}A_{\text {osc }} & A_{\text {osc }} & A_{\text {osc }} & A_{\text {osc }}\end{array}\right]^{T}$.

- The experiments were conducted in two stages. In the first stage the following two experimental scenarios have been carried out to compare the linear PID controller with the nonlinear RISE feedback controller: (i) Control in the nominal case, (ii) External disturbance rejection. Then, in the second stage, the best controller (according to the first stage) among the two proposed controllers will be tested in the third experimental scenario (iii) Robustness towards uncertainties in the parameters (buoyancy change).

- All the proposed controllers were implemented on the robot using ROS (Robot Operating System).

- All the relevant real-time data were saved to ROS log files (rosbags). Using rosbags enables inspecting the data exactly in the same form as used by the robot software. The rosbag files were then downloaded from the robot after each experiment.

- It is worth noting that the weather conditions were not favorable (presence of environmental disturbances such as wind, current, surface waves, etc).

\section{B. Experimental results}

1) Scenario 1 : Control in nominal case: The obtained results (in terms of trajectory tracking as well as tracking error) are depicted in Figs. 4 and 5. According to these two
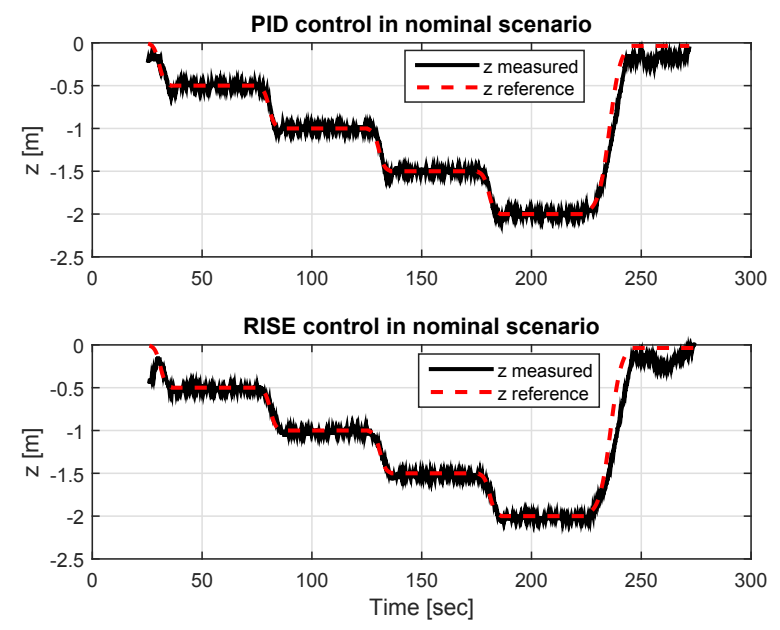

Fig. 4. Depth tracking for nominal case. Top: The PID controller, bottom: RISE controller.
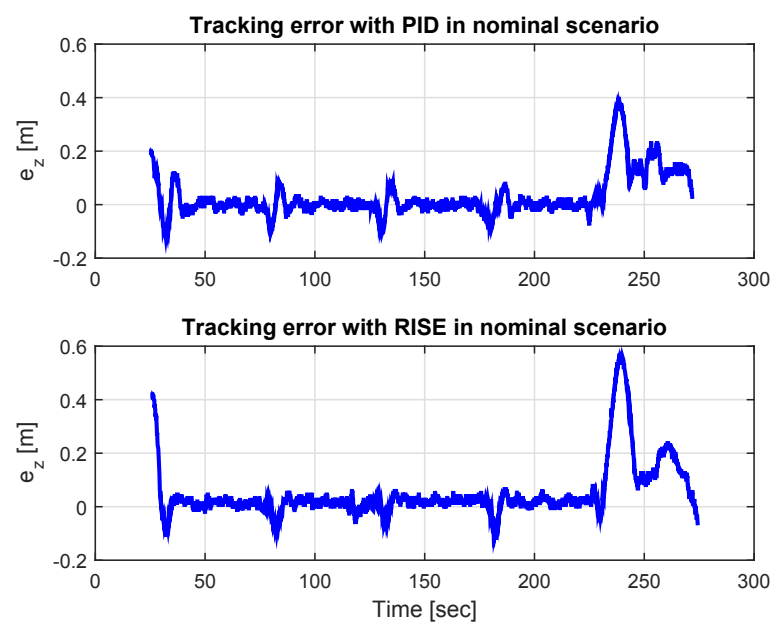

Fig. 5. Evolution of depth tracking errors versus time for nominal case. Top: For the PID controller, bottom: For RISE controller.

figures, it can be clearly observed that both controllers are able to steer the system output in order to track the desired trajectory. The computation of the Root Mean Square (RMS) error may be a good quantitative criterion to compare both controllers. The evaluation of this criterion gives the values $2.9683 \times 10^{-2}$ (for the PID) and $2.8648 \times 10^{-2}$ (for RISE controller). The tracking performance of RISE controller is consequently better than the one of the PID controller. It is 
worth to note, for both controllers, that at the beginning and at the end of each depth experiment, the robot is very close to the surface, and consequently subject to highly disturbing waves and currents. For both controllers, in the beginning and at the end of each depth experiment, the robot is very close to the surface subjected to highly disturbing waves and currents, which is also reflected in higher tracking errors. The evolution of the roll and pitch angles of the robot are depicted in Fig. 6, where one can observe that they remain most of the time around zero, except when the robot arrives to the surface.
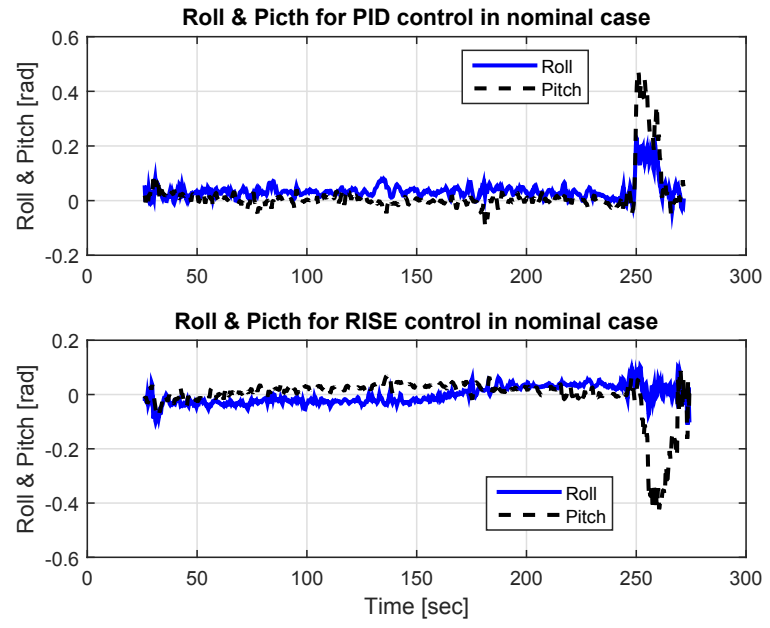

Fig. 6. Evolution of pitch and roll angles versus time. Top: For the PID controller, bottom: For RISE controller.

2) Scenario 2 : External disturbances rejection: The experimental procedure consists of tracking of the desired reference trajectory moving from the surface to the desired constant depth, followed by a steady state value at this desired depth, then moving back to the surface. The disturbance consists of lifting the robot to the surface during the steady state phase to test weather the controllers are able to reject this external disturbance. The obtained results, with both controllers, for this scenario are depicted in Fig. 7, for the reference tracking; and in Fig. 8, for the tracking errors. In the case of the PID controller, the external disturbance is applied around at $53 \mathrm{sec}$. Despite of the big value of the disturbance, the controller is able to steer the robot back to the reference trajectory with a recovery time of approximately $10 \mathrm{sec}$. The stability of the closed-loop system is preserved despite of the big amplitude of the external disturbance. In the case of RISE controller, two external disturbances have been applied to the robot: The first one around approximately $57 \mathrm{sec}$ and the second one around 77 sec. According to Figs. 7 and 8 , the controller is able to reject these external disturbances and bring the robot back around its steady state value in a very stable way.

To sum up for this scenario, the tracking performance of the RISE controller is better than the one of the PID, even though both controllers guarantee a recovery after the applied external disturbances. Indeed, more oscillations
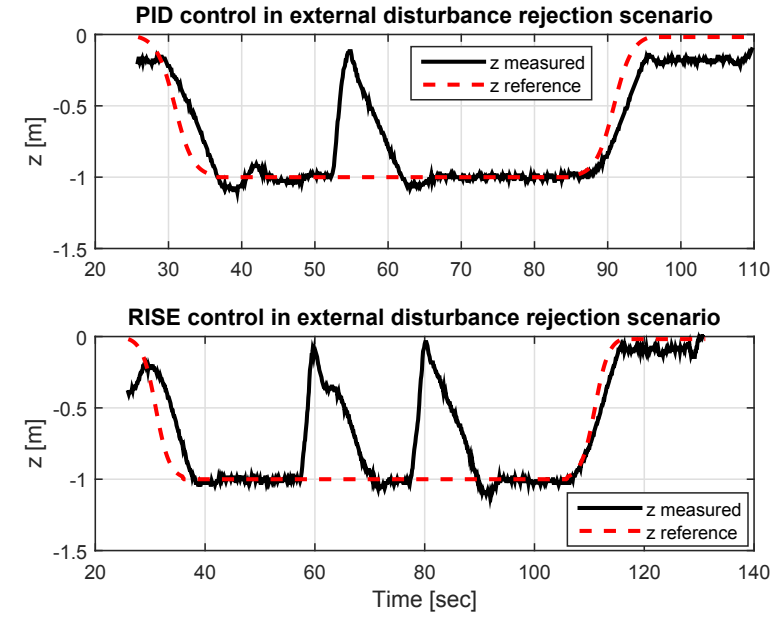

Fig. 7. Depth tracking for external disturbance rejection case. Top: The PID controller, bottom: RISE controller.
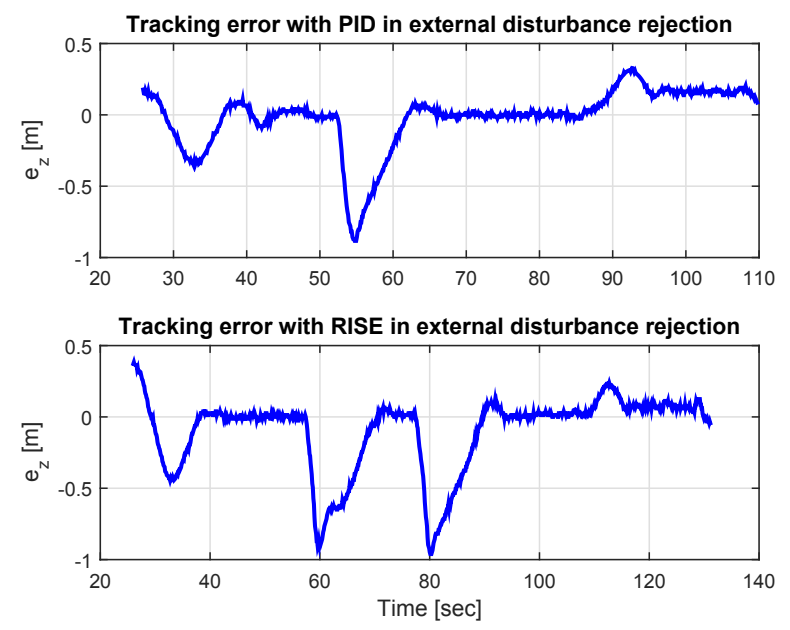

Fig. 8. Evolution of depth tracking errors versus time for the external disturbance rejection case. Top: The PID controller, bottom: RISE controller.

can be noticed around $38 \mathrm{sec}$ for the case of the PID; whereas, no oscillations for RISE controller. Furthermore, when the robot moves back to the surface, it can also be noticed that the tracking error for the case of the PID is bigger than the case of the RISE controller. Consequently, the RISE controller outperforms the PID controller. In the following the robustness of the former is shown through an experimental scenario involving a change in one dynamic parameter of the system, namely the buoyancy.

3) Scenario 3 : Robustness towards buoyancy change: In this experimental scenario, the idea lies in introducing an uncertainty on one of the parameters of the robot (the buoyancy) and to check weather the RISE controller is able to compensate for this uncertainty. Fig. 9 illustrates how the buoyancy of the robot has been modified. All the parameters of the controller have been kept the same as for the previous experimental scenarios. The obtained results for this scenario are shown in Fig. 10, representing the depth 


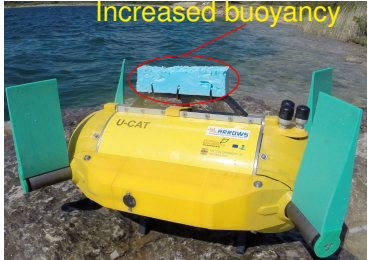

Fig. 9. Illustration of the case of increased buoyancy: The piece of float fixed to the body of the robot will increase its buoyancy.

trajectory tracking as well as the evolution of the tracking error. According to the obtained results one can notice that the RISE controller, despite this uncertainty, is able to track the reference trajectory. The tracking performance is slightly degraded, but still acceptable. Indeed, except of the immersion of the robot, the tracking error is close to zero. One can conclude that the RISE controller is robust towards this uncertainty of buoyancy of the robot.
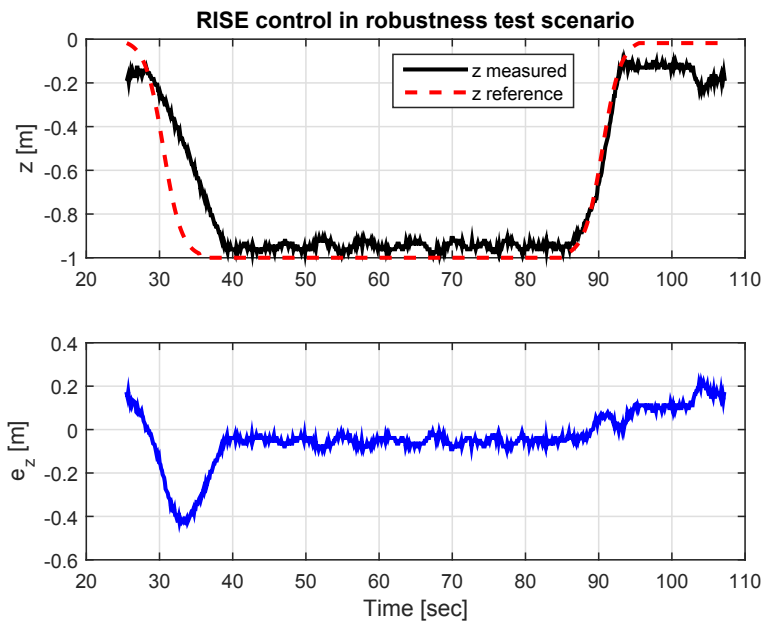

Fig. 10. Depth tracking for robustness towards uncertainties (RISE controller). Top: Trajectory tracking, bottom: Evolution of the tracking error.

\section{CONCLUSIONS AND FURTHER WORK}

In this work, automatic depth control of U-CAT biomimetic underwater AUV was studied. Two depth controllers have been proposed, implemented and tested in real operating conditions. Namely a PID controller and a nonlinear RISE feedback controller. Both controllers have been tested on the robot in real operating conditions (Rummu lake in Estonia). The nonlinear RISE feedback controller outperforms the PID controller in terms of tracking performance. Although the obtained results are satisfactory, several issues remain to be investigated in future work. For instance, one could consider more advanced controllers, such as modelbased or robust and adaptive controllers. Furthermore, the consideration of control of multiple degrees of freedom (yaw and depth for instance) should also be considered.

\section{REFERENCES}

[1] J. S. Palmisano and et al., "A comprehensive allometric analysis of bio-mimetic mpf-type uuvs," in 18th International Symposium on Unmanned Untethered Submersible Technology, Portsmouth, $\mathrm{NH}$ USA, 2013.

[2] A. Konno and et al., "Development of turtle-like submergence vehicle," in 7th International Symposium on Marine Engineering, Tokyo, Japan, 2005.

[3] M. Kemp and et al., "Madeleine: an agile auv propelled by flexible fins," in 14th International Symposium on Unmanned Untethered Submersible Technology, Durham, New Hampshire, USA, 2005.

[4] S. C. Licht, Biomimetic oscillating foil propulsion to enhance underwater vehicle agility and maneuverability. DTIC Document, 2008.

[5] S. Licht and N. Durham, "Biomimetic robots for environmental monitoring in the surf zone \& in very shallow water," in IEEE/RSJ International Conference on Intelligent Robots and Systems, Vila Moura, Algarve, Portugal, 2012.

[6] G. G. Dudek and et al., "Aqua: An amphibious autonomous robot," in Computer, 2007.

[7] N. Plamondon and M. Nahon, "Adaptive controller for a biomimetic underwater vehicle," Journal of Unmanned Vehicle Systems, vol. 1, pp. 1-13, 2013.

[8] J. D. Geder and et al., "Maneuvering performance of a four-fin bioinspired uuv," in Oceans-San Diego, 2013.

[9] J. S. Palmisano and et al., "Robotic pectoral fin thrust vectoring using weighted gait combinations," Applied Bionics and Biomechanics, vol. 9, pp. 333-345, 2012.

[10] _ - "Design of a biomimetic controlled-curvature robotic pectoral fin," in IEEE International Conference on Robotics and Automation, 2007

[11] H. Kim, S.-H. Song, and S.-H. Ahn, "A turtle-like swimming robot using a smart soft composite (ssc) structure," Smart Materials and Structures, vol. 22, no. 1, p. 014007, 2013.

[12] B. Allotta and et al., "The arrows project: adapting and developing robotics technologies for underwater archaeology," in IFAC workshop on navigation and control of underwater vehicles (NGCUV 2015), Girona, Spain, 2015.

[13] J. Yuh, "Design and control of autonomous underwater robots: A survey," Autonomous Robots, vol. 8, 2000.

[14] G. Yao and et al., "Development of a turtle-like underwater vehicle using central pattern generator," in IEEE International Conference on Robotics and Biomimetics (ROBIO), Shenzen, China, 2013, pp. 44-49.

[15] T. Salumae, R. Raag, J. Rebane, A. Ernits, G. Toming, M. Ratas, and M. Kruusmaa, "The arrows project: adapting and developing robotics technologies for underwater archaeology," in IEEE Oceans-St. John's, 2014.

[16] T. Fossen, Marine Control Systems:Guidance, Navigation and Control of Ships, Rigs and Underwater Vehicles. As,Trondheim: Marine Cybernetics, 2002.

[17] W. Wang, PID Controller Design Approaches - Theory, Tuning and Application to Frontier Areas, M. Vagia, Ed. InTech, 2012.

[18] N. Fischer, D. Hughes, P. Walters, E. M. Schwartz, and W. E. Dixon, "Nonlinear rise-based control of an autonomous underwater vehicle," IEEE Transactions on Robotics, vol. 30, no. 4, pp. 845-852, 2014.

[19] B. Xian, D. M. Dawson, M. S. D. Queiroz, and J. Chen, "A continuous asymptotic tracking control strategy for uncertain multi-input nonlinear systems," vol. 49, no. 7, pp. 1206-1211, Jul. 2004.

[20] P. M. Patre, W. MacKunis, C. Makkar, and W. E. Dixon, "Dynamics and control of redundantly actuated parallel manipulators," vol. 16, no. 2, pp. 473-379, Mar. 2008.

[21] P. M. Patre, W. MacKunis, K. Kaiser, and W. E. Dixon, "Asymptotic tracking for uncertain dynamic systems via a multilayer neural network feedforward and rise feedback control structure," vol. 53, no. 9, pp. 2180-2185, Oct. 2008.

[22] M. Taktak, A. Chemori, J. Ghommam, and N. Derbel, "Rise feedback control for a $\mathrm{r} / \mathrm{w}$ head track following in hard disk drives," in Proc. Multi-Conference on Systems, Signals and Devices (SSD'14), Barcelona, Spain, 2014.

[23] N. Fischer, S. Bhasin, and W. Dixon, "Nonlinear control of an autonomous underwater vehicle: A rise-based approach," in Proc. IEEE American Control Conference (ACC'11), San Francisco, USA, Jun. 2011, pp. 3972-3977.

[24] M. Bennehar, A. Chemori, and F. Pierrot, "A novel rise-based adaptive feedforward controller for redundantly actuated parallel manipulators," in Proc. IEEE International Conference on Intelligent Robots and Systems (IROS'14), Chicago, Illinois, USA, 2014, pp. 2389-2394. 